ORIGINAL RESEARCH

\title{
Circadian and Sex Differences After Acute High-Altitude Exposure: Are Early Acclimation Responses Improved by Blue Light?
}

Juan A. Silva-Urra, GP, MSc; Cristian A. Núñez-Espinosa, MSc; Oscar A. Niño-Mendez, MSc; Héctor Gaitán-Peñas, MSc; Cesare Altavilla, MSc; Andrés Toro-Salinas, MSc; Joan R. Torrella, PhD; Teresa Pagès, PhD; Casimiro F. Javierre, MD; Claus Behn, MD; Ginés Viscor, PhD

From the Departamento Biomédico, Universidad de Antofagasta, Antofagasta, Chile (Mr Silva-Urra); the Departament de Fisiologia $i$ Immunologia (Mr Núñez-Espinosa, Mr Gaitán-Peñas, Mr Altavilla, Mr Toro-Salinas, and Drs Torrella, Pagès, and Viscor); and Departament de Ciències Fisiològiques II, (Mr Niño-Mendez and Dr Javierre), Universitat de Barcelona, Barcelona, Spain; and the Laboratorio de Ambientes Extremos (ICBM), Universidad de Chile, Santiago de Chile, Chile (Dr Behn).

Objective.-The possible effects of blue light during acute hypoxia and the circadian rhythm on several physiological and cognitive parameters were studied.

Methods.-Fifty-seven volunteers were randomly assigned to 2 groups: nocturnal (2200-0230 hours) or diurnal (0900-1330 hours) and exposed to acute hypoxia (4000 m simulated altitude) in a hypobaric chamber. The participants were illuminated by blue LEDs or common artificial light on 2 different days. During each session, arterial oxygen saturation $\left(\mathrm{Spo}_{2}\right)$, blood pressure, heart rate variability, and cognitive parameters were measured at sea level, after reaching the simulated altitude of $4000 \mathrm{~m}$, and after 3 hours at this altitude.

Results.-The circadian rhythm caused significant differences in blood pressure and heart rate variability. A $4 \%$ to $9 \%$ decrease in waking nocturnal $\mathrm{Spo}_{2}$ under acute hypoxia was observed. Acute hypoxia also induced a significant reduction $(4 \%-8 \%)$ in systolic pressure, slightly more marked (up to $13 \%)$ under blue lighting. Women had significantly increased systolic (4\%) and diastolic (12\%) pressures under acute hypoxia at night compared with daytime pressure; this was not observed in men. Some tendencies toward better cognitive performance (d2 attention test) were seen under blue illumination, although when considered together with physiological parameters and reaction time, there was no conclusive favorable effect of blue light on cognitive fatigue suppression after 3 hours of acute hypobaric hypoxia.

Conclusions.- It remains to be seen whether longer exposure to blue light under hypobaric hypoxic conditions would induce favorable effects against fatigue.

Key words: altitude shift work, intermittent hypoxia, altitude acclimatization, heart rate variability, attentional blink, blue light illumination

\section{Introduction}

Exposure to altitude inflicts many environmental stresses on the human body derived from factors such as low temperatures, high doses of solar and ionizing radiation, strong winds, and low absolute humidity. ${ }^{1}$ However, most of the problems that occur at high altitude are attributable to the hypobaric hypoxia caused by the decrease in atmospheric pressure.

Corresponding author: Ginés Viscor, Departament de Fisiologia i Immunologia, Universitat de Barcelona, Avenida Diagonal, 643, E08023 Barcelona, Spain (e-mail: gviscor@ub.edu).
Thousands of workers in the Americas and Central Asia routinely perform activities requiring periods of a few hours at high altitudes followed by a return to low altitudes or even to sea level on a shift-work model (mining, transport, astronomical facilities, etc.), or rapid ascent and descent to moderate and even high altitudes (rescue, defense, etc.). Because of the short duration of the latter type of exposure, workers rarely present severe symptoms of acute mountain sickness (AMS) as a consequence of their exposure to hypobaric hypoxia. However, the effects of hypoxia on some organs involved in the execution of complex tasks (ie, brain, peripheral 
nerves, and even the heart, which performs brain perfusion) cannot be neglected. Acute hypoxia exposure may manifest early in terms of cognitive or alertness parameters, potentially altering proficiency at complex tasks. The temporary and mild character of these alterations, and their spontaneous resolution in most cases, do not contribute to a proper evaluation of the reduced performance and productivity loss during the first hours or days of altitude exposure. Previous reports have shown a decrease in physical capacity associated with short periods of altitude exposure ${ }^{2,3}$ and deleterious effects on neurobehavioral responses ${ }^{4,5}$ that could increase the risk of work-related accidents, especially for those commuting between places at different altitudes. All these factors may contribute to a high rate of transience in the workforce at high altitude because of intolerance to hypobaric hypoxia. ${ }^{6}$ Moreover, the incidence of vehicle rollover accidents (especially during descent) may be exacerbated by hypoxia because of a decrease in perception, mental concentration, and reaction speed, resulting in a reduced ability to drive vehicles or control heavy machinery. ${ }^{7}$ Recent research by our group revealed a high frequency of cardiac arrhythmic events during car descents from $5050 \mathrm{~m}$ to $2950 \mathrm{~m}^{8}$

As a consequence, some countries have developed laws to protect workers at altitude. In Chile, since December 2012, legislation recognizes disorders resulting from hypobaria and requires companies operating at altitude to provide adequate education and preventive measures for their workers. To mitigate these problems, some strategies have been proposed for high-altitude working facilities, such as 1) the installation of base camps at lower altitudes for sleep and leisure ${ }^{9-11}$;2) the use of physiological preacclimation strategies, such as hypobaric chambers ${ }^{12}$; 3) the use of portable canned oxygen for pulse delivery, as used, for example, by visitors to the ALMA telescope site $(5050 \mathrm{~m})$; or 4$)$ oxygen enrichment of workplaces. ${ }^{13-16}$

The application of blue light illumination has proved to be a very effective way of improving the regulation of circadian rhythms. ${ }^{17-20}$ In this context and in the search for new preventive strategies to optimize human labor at altitude, the idea has emerged that blue light could have favorable effects at high altitude. For this reason, our group recently tested blue LED installations in the driver cabins of trucks used for the transportation of mining material for their effects on driver alertness during the night (Behn et al, unpublished data). The physiological basis of this methodology is derived from studies in mammals that demonstrate that the retinohypothalamic tract is involved in the regulation of circadian rhythms by light. $^{21}$ The final step in the regulation loop is the production and rhythmic secretion of melatonin from the pineal gland via a circuit that allows for the light-dark cycle to be sensed. The circuit involves the activity of the so-called intrinsically photosensitive retinal ganglion cells of the retina. ${ }^{22}$ These cells are projected to the suprachiasmatic nucleus ${ }^{23}$ and ultimately have afferent connections to the pineal gland, where melatonin is produced. $^{24,25}$ The secretion of melatonin can be inhibited by light, but not all wavelengths inhibit it. Wavelengths above $530 \mathrm{~nm}$ have no suppressive effects, whereas the blue band (wavelengths between 446 and $477 \mathrm{~nm}$ ) has been found to be the optimal range for melatonin inhibition. ${ }^{17}$ Exposure to blue light has several physiological effects aside from the suppression of melatonin secretion, including increases in body temperature, alterations of cognitive performance, and fatigue during the night. ${ }^{26}$ Moreover, melatonin has been experimentally demonstrated to increase the ventilatory response to hypoxia. ${ }^{27}$ On the other hand, it has been reported that color vision can be adversely affected at high altitude, especially discrimination along the tritan (blue) axis, ${ }^{28}$ which could be related to abnormal melatonin release and wakeful status under such conditions.

Our hypothesis was that blue light decreases the state of drowsiness, presumably by reducing the secretion of melatonin, especially at night, and therefore improves cognitive performance indicators. The main objective of this study was to detect, by means of measuring noninvasive physiological parameters and psychological testing, the signs of cognitive fatigue and poor acclimatization to high altitude conditions, and to evaluate the possible positive effect of blue light in the early phase of accommodation. Thus, we tested the possible alterations of mental concentration and alertness of subjects under hypobaric hypoxic conditions in daytime and nighttime scenarios.

\section{Methods}

\section{SUBJECTS}

This study was conducted in accordance with the Helsinki Declaration concerning the ethical principles of human experimentation. A total of 57 healthy volunteers (25 men and 32 women) were randomly selected from a group of college students. Table 1 shows the anthropometric data of the participants in this study. They were initially given a general information session and personal interview. All subjects underwent a baseline hypoxia tolerance test and were asked not to ingest alcohol and caffeine for 12 hours before each session of the study.

\section{LIGHTING}

The lighting level inside the hypobaric chamber was measured using a light meter (PCE 172; PCE Instruments, 
Table 1. Select characteristics of the study participants

\begin{tabular}{lcc}
\hline Characteristic & Men $(n=25)$ & Women $(n=32)$ \\
\hline Age (years) & $23.8 \pm 4.2$ & $22.0 \pm 3.5$ \\
Weight $(\mathrm{kg})$ & $71.4 \pm 9.8$ & $57.8 \pm 7.4$ \\
Height $(\mathrm{cm})$ & $175 \pm 8$ & $166 \pm 6$ \\
BMI $\left(\mathrm{kg} / \mathrm{m}^{2}\right)$ & $23.2 \pm 2.7$ & $20.9 \pm 2.4$ \\
$\%$ Smokers & 16.0 & 18.8 \\
\hline
\end{tabular}

Values are reported as mean $\pm \mathrm{SD}$.

BMI, body mass index.

Jupiter, FL) and followed the guidelines established by the European Union for workplace lighting. To meet the recommendations, the incident light was adjusted at 3 different points to $30 \mathrm{lux}$ at $20 \mathrm{~cm}$ from the edge of the working table, ensuring uniform lighting conditions inside the hypobaric chamber. Measurements were performed under 2 experimental lighting conditions: 1) normal artificial lighting (NAL) produced by 5 "incandescent-like" (380-750 nm) soft-white halogen roof lamps $(2700 \mathrm{~K})$; and 2) blue light (bLED) produced by 2 strips of narrow-wavelength $(465-475 \mathrm{~nm})$ blue LEDs placed along the roof of the chamber. According to the literature, this is within the optimal range for effects on humans. ${ }^{29-31}$

\section{HYPOXIA EXPOSURE}

The participants were scheduled for 2 simulated altitude exposure sessions on different days in a hypobaric chamber located at the Bellvitge Health Campus of the University of Barcelona. They were randomly assigned to 1 of 2 groups: nocturnal (11 men and 19 women) or diurnal (14 men and 13 women). The experimental schedule for the nocturnal group was from 2200 to 0230 hours; the diurnal schedule was from 0900 to 1330 hours. A small group (5-6 subjects) took part in each session. At the first session, a full medical examination, including medical history and physical characteristics, was performed. The subjects were allocated to their posts within the hypobaric chamber, and the first set of measurements was performed.

Cardiovascular parameters (heart rate, blood pressure, and arterial oxygen saturation $\left[\mathrm{Spo}_{2}\right]$ ) were measured before each set of cognitive and heart rate variability (HRV) tests. Once finished, the door of the hypobaric chamber was closed and simulated altitude exposure commenced. The simulated ascent to $4000 \mathrm{~m}$ took 20 minutes; simulated altitude lasted for 3 hours, and the final repressurization period was 20 minutes. Either NAL or bLED was used as the only source of illumination in each session. The order of the type of light was randomly assigned to each group to avoid any psychological bias. All the parameters studied were obtained under 3 different conditions: at sea level (A0); 15 minutes after arrival at a simulated altitude of $4000 \mathrm{~m}$ (A1); and after 3 hours of simulated altitude exposure (A2).

\section{MEASUREMENT PROTOCOL}

The subjects were at rest and silent for 10 minutes during heart rate monitoring for subsequent HRV analysis. All subjects then proceeded to complete 2 tests to measure concentration and mental fatigue. First, all the subjects performed the $\mathrm{d} 2$ attention test together. ${ }^{32}$ After a short period of relaxation, the attentional blink test ${ }^{33}$ was performed.

\section{ANALYSIS OF HEART RATE VARIABILITY}

Heart rate variability was measured by means of a Polar Team2 Pro System (Polar Electro Inc, Lake Success, NY). This system has several Team2 transmitters, which send the signal to the receiver unit via Bluetooth technology, thus allowing simultaneous monitoring of several subjects. Beat-to-beat heart rate (R-R interval) was recorded for each individual for at least 5 minutes. The data were analyzed by means of Kubios software (University of Eastern Finland, Kuopio, Finland). ${ }^{34} \mathrm{We}$ evaluated variations of HRV by time and frequency domain methods. In the time domain methods, we analyzed the following statistical measurements: standard deviation of all R-R intervals (SDNN); the square root of the mean squared differences of successive R-R intervals (RMSSD), expressed in milliseconds; and the proportion of heart beats with a duration $50 \mathrm{~ms}$ longer or shorter than the successive interval (pNN50), expressed as a percentage. We also analyzed the areas of the spectral peaks of very-low-frequency (VLF), lowfrequency (LF), and high-frequency (HF) components expressed as a percentage. The LF/HF ratio, for analysis of the sympathetic-parasympathetic balance, was also calculated. The power spectrum density was estimated using fast Fourier transformations.

\section{D2 ATTENTION TEST}

For several years, the $\mathrm{d} 2$ attention test has been one of the most useful tools for assessing concentration and selective attention. It assesses the control of attention, error rate, work rate, and concentration fluctuation. In this study, we used the Spanish adaptation. ${ }^{35}$

Before the test, a clear explanation was given and a practice test was performed. The test consists of 14 lines of the letters "d" and " $p$ " in Courier font accompanied by a variable number (up to 4 ) of small dashes in subscript 
or superscript positions. There is a time limit of 20 seconds per line to be resolved. The respondent is required to scan the lines and cross out all occurrences of the letter "d" with 2 dashes, while ignoring all other characters. The $\mathrm{d} 2$ test design allows 3 components reflecting attention behavior to be identified: the rate or amount of work, the degree of precision in performing the task, and the relationship between these factors. Analyzing the scores from this test, conclusions can be drawn about the degree of activity, the stability and consistency in the task, and fatigue and attentional inhibition efficiency. ${ }^{35}$ In this study, 2 main parameters obtained from the $\mathrm{d} 2$ test were considered: the total effectiveness (TOT) was calculated as the total number of items processed minus error scores, and the concentration index $(\mathrm{CON})$ was calculated as the difference between correct and incorrect answers.

\section{ATTENTIONAL BLINK TEST}

The attentional blink $(\mathrm{AB})$ test was first described by Raymond et al. ${ }^{33}$ The $\mathrm{AB}$ test is based on the assumption that there is a known decline in visual performance during task execution. This is attributable to the consumption of resources by other concurrent tasks, when the 2 are out of synchronization. This phenomenon is observed when using the paradigm of rapid serial visual presentation (RSVP): When a series of visual stimuli is presented in rapid succession in the same spatial location, subjects often fail to detect the second target when it is presented between 200 and $500 \mathrm{~ms}$ after the first one. The RSVP is often used to study visual perception, both in psychophysics and cognitive psychology, ${ }^{36}$ and in this case it is used to establish whether there is a decline in visual perception and thus in the nervous system that controls the body.

In this test, 2 condition responses were used: simple and dual tasks. In the simple task, participants were required to indicate at the end of each visual representation series of letters, without any time limit, whether a target stimulus (the letter "X") appeared, which occurred in $50 \%$ of the series. In the double response task, subjects were asked to detect 2 different targets, for example, whether a letter "O" appeared after an "X." In this second task, it becomes clear that performance is out of the range expected by chance. The software automatically computed the number of right answers (RA) and the reaction time (RT, expressed as $\times 100 \mathrm{~ms}$ ) in the second task.

\section{STATISTICAL ANALYSIS}

The data were analyzed using SigmaPlot 12 software (Systat Software Inc, San Jose, CA). Multiple comparisons were performed by 3-way analysis of variance (3-way ANOVA) with the factors L (light, with levels bLED and NAL), H (hypoxia, with levels A0, A1, and A2), and S (schedule, with levels day and night). The power analysis for the ANOVA test was automatically performed according to the following adjustments: minimum detectable difference in means of 0.8 , expected standard deviation of residuals at 0.3 , and alpha of 0.05 . Holm-Sidak and paired $t$ tests were used as post-hoc analysis for pairwise comparisons within levels. Differences were considered statistically significant when the probability value was $<.05$. Unless otherwise indicated, values are expressed as mean $\pm \mathrm{SD}$.

\section{Results}

Mean data and SD for all physiological parameters are presented in Table 2 (men) and Table 3 (women), and cognitive parameters are given in Table 4. Statistical significance for the interaction (3-way ANOVA) between the 3 factors in the experimental design (light, hypoxia, and schedule) is presented in the corresponding columns on the left. Significant differences after the post-hoc analysis are shown as superscripts according to the code explained in the footnotes of each table.

\section{PHYSIOLOGICAL PARAMETERS}

As expected, hypoxia was followed by a marked reduction in $\mathrm{SpO}_{2}$. In both men and women, this decrease in $\mathrm{SpO}_{2}$ was significantly pronounced at night under simulated altitude conditions (A1 and A2) in comparison to daytime. This finding was not evident under normoxic conditions, and no effect was observed regarding lighting conditions.

Blood pressure behaved differently depending on sex. In men there were no significant differences in systolic and diastolic pressures between the day and night schedules (Table 2). However, in women significant increases of systolic pressure were observed at night at $\mathrm{A} 0$ with bLED and at $\mathrm{A} 1$ under both lighting conditions, and for diastolic pressure in all cases (Table 3). Moreover, hypoxia induced a significant reduction in systolic pressure (4\%-8\%), which was slightly more marked under bLED lighting (4\%-13\%). This effect of hypoxia was not observed for diastolic pressure.

Heart rate was always higher at night and also, as expected, under the 2 hypoxic conditions studied. Regarding HRV, some differences again appeared depending on sex. SDNN was significantly higher during the night in men but not in women. Nevertheless, women showed a significant reduction of this parameter at night at A2 under both lighting conditions, and a significant reduction in RMSSD and pNN50 was also evident in women at night under all conditions. VLF and HF 
Table 2. Physiological parameters in men exposed to 2 kinds of light under normobaric normoxic and hypobaric hypoxic conditions

\begin{tabular}{|c|c|c|c|c|c|c|c|c|c|c|c|c|c|c|c|}
\hline \multirow[b]{3}{*}{ Variable } & & & & \multicolumn{6}{|c|}{$b L E D$} & \multicolumn{6}{|c|}{$N A L$} \\
\hline & \multicolumn{3}{|c|}{ 3-way ANOVA } & \multicolumn{2}{|c|}{$A O$} & \multicolumn{2}{|c|}{$A 1$} & \multicolumn{2}{|c|}{$A 2$} & \multicolumn{2}{|c|}{$A O$} & \multicolumn{2}{|c|}{$A 1$} & \multicolumn{2}{|c|}{$A 2$} \\
\hline & $L$ & $H$ & $S$ & Day & Night & Day & Night & Day & Night & Day & Night & Day & Night & Day & Night \\
\hline $\mathrm{SpO}_{2}(\%)$ & NS & $b c$ & $e$ & $99 \pm 1$ & $98 \pm 1$ & $83 \pm 5^{f}$ & $74 \pm 5^{f h}$ & $82 \pm 4^{f}$ & $75 \pm 5^{f h}$ & $99 \pm 1$ & $98 \pm 1$ & $84 \pm 6^{f}$ & $75 \pm 6^{f h}$ & $81 \pm 3^{f}$ & $77 \pm 6^{f h}$ \\
\hline HR (bpm) & NS & $b c$ & $e$ & $71 \pm 8$ & $79 \pm 12^{h}$ & $77 \pm 8^{f}$ & $90 \pm 18^{f h}$ & $76 \pm 6^{f}$ & $89 \pm 17^{h}$ & $69 \pm 9$ & $76 \pm 9^{h}$ & $76 \pm 8^{f}$ & $86 \pm 13^{f h}$ & $74 \pm 10^{f}$ & $87 \pm 17^{f h}$ \\
\hline $\mathrm{SP}(\mathrm{mm} \mathrm{Hg})$ & NS & $b c d$ & NS & $132 \pm 10$ & $136 \pm 13$ & $123 \pm 10^{f}$ & $130 \pm 13^{f}$ & $115 \pm 15^{f g}$ & $121 \pm 13^{f g}$ & $129 \pm 9$ & $133 \pm 16$ & $122 \pm 14^{f}$ & $127 \pm 20$ & $118 \pm 10^{f}$ & $121 \pm 14^{f}$ \\
\hline $\begin{array}{l}\text { DP }(\mathrm{mm} \mathrm{Hg}) \\
\text { HRV }\end{array}$ & NS & NS & NS & $77 \pm 7$ & $74 \pm 6$ & $74 \pm 11$ & $72 \pm 10$ & $70 \pm 10$ & $69 \pm 7$ & $76 \pm 6$ & $79 \pm 18$ & $72 \pm 8$ & $73 \pm 17$ & $72 \pm 9$ & $77 \pm 18$ \\
\hline SDNN & $a$ & NS & $e$ & $59 \pm 23$ & $76 \pm 31^{h}$ & $61 \pm 20$ & $73 \pm 28^{h}$ & $59 \pm 14$ & $64 \pm 22^{h}$ & $59 \pm 16$ & $88 \pm 31^{h}$ & $60 \pm 17$ & $73 \pm 26^{h}$ & $59 \pm 18$ & $81 \pm 29^{h i}$ \\
\hline RMSSD (ms) & NS & NS & NS & $49 \pm 28$ & $50 \pm 26$ & $46 \pm 23$ & $42 \pm 20$ & $40 \pm 13$ & $39 \pm 10$ & $48 \pm 20$ & $70 \pm 31$ & $49 \pm 20$ & $45 \pm 29$ & $35 \pm 14$ & $55 \pm 23$ \\
\hline pNN50 (\%) & NS & $c$ & NS & $27 \pm 20$ & $24 \pm 18$ & $17 \pm 11^{f}$ & $17 \pm 15^{f}$ & $15 \pm 9^{f}$ & $15 \pm 13^{f}$ & $28 \pm 17$ & $32 \pm 21$ & $20 \pm 12$ & $23 \pm 17$ & $13 \pm 10^{f g}$ & $20 \pm 15$ \\
\hline VLF (\%) & NS & NS & $e$ & $20 \pm 15$ & $34 \pm 19$ & $25 \pm 12$ & $31 \pm 18$ & $21 \pm 12$ & $30 \pm 18$ & $22 \pm 12$ & $22 \pm 14$ & $24 \pm 10$ & $26 \pm 17$ & $27 \pm 16$ & $37 \pm 16^{h}$ \\
\hline $\mathrm{LF}(\%)$ & NS & $c d$ & NS & $43 \pm 17$ & $42 \pm 19$ & $48 \pm 9$ & $53 \pm 19$ & $61 \pm 16^{f g}$ & $54 \pm 19$ & $46 \pm 18$ & $47 \pm 19$ & $45 \pm 14$ & $49 \pm 23$ & $57 \pm 17^{f g}$ & $51 \pm 19$ \\
\hline $\mathrm{HF}(\%)$ & NS & $c d$ & $e$ & $38 \pm 22$ & $25 \pm 14^{h}$ & $27 \pm 12^{f}$ & $17 \pm 5^{h}$ & $18 \pm 9^{f g}$ & $16 \pm 6$ & $32 \pm 19$ & $30 \pm 16$ & $32 \pm 17$ & $25 \pm 20$ & $16 \pm 8^{f g}$ & $14 \pm 8$ \\
\hline $\mathrm{LF} / \mathrm{HF}$ & NS & NS & NS & $2.9 \pm 3.6$ & $2.7 \pm 3.1$ & $3.4 \pm 4.3$ & $2.9 \pm 1.0$ & $4.9 \pm 4.1$ & $4.3 \pm 4.8$ & $3.2 \pm 4.6$ & $2.4 \pm 1.9$ & $2.3 \pm 2.5$ & $2.4 \pm 2.0$ & $4.6 \pm 2.9$ & $5.0 \pm 3.8$ \\
\hline
\end{tabular}

All data are mean \pm SD. Multiple comparisons were performed by a 3-way analysis of variance (3-way ANOVA) with factors L (light, with levels bLED and NAL), H (hypoxia, with levels A0, $\mathrm{A} 1$, and $\mathrm{A} 2$ ), and $\mathrm{S}$ (schedule, with levels day and night). Holm-Sidak and paired $t$ tests were used in post-hoc analysis for pairwise comparisons within levels.

bLED, blue LED; NAL, normal artificial light; A0, at sea level; A1, 15 minutes after ascent to simulated altitude of $4000 \mathrm{~m}$; A2, 3 hours of simulated altitude of $4000 \mathrm{~m}$; Spo 2 , arterial saturation; NS, not significant; HR, heart rate; SP, systolic blood pressure; DP, diastolic blood pressure; HRV, heart rate variability; SDNN, standard deviation of R-R intervals; RMSSD, square differences of successive R-R intervals; pNN50, differences of successive intervals greater than $50 \mathrm{~ms}$; VLF, very low frequency; LF, low frequency; HF, high frequency.

${ }^{a}$ bLED vs NAL, $P<.05$, 3-way ANOVA.

${ }^{b} \mathrm{~A} 0$ vs $\mathrm{A} 1, P<.05,3$-way ANOVA.

${ }^{c}$ A0 vs A2, $P<.05$, 3-way ANOVA.

${ }^{d} \mathrm{~A} 1$ vs A2, $P<.05,3$-way ANOVA.

$e$ day vs night, $P<.05,3$-way ANOVA.

$f$ vs A0, $P<.05$, Holm-Sidak and paired $t$ tests.

${ }^{g}$ vs A1, $P<.05$, Holm-Sidak and paired $t$ tests.

${ }^{h}$ vs day, $P<.05$, Holm-Sidak and paired $t$ tests.

${ }^{i}$ vs bLED, $P<.05$, Holm-Sidak and paired $t$ tests 
Table 3. Physiological parameters in women exposed to 2 kinds of light under normobaric normoxic and hypobaric hypoxic conditions

\begin{tabular}{|c|c|c|c|c|c|c|c|c|c|c|c|c|c|c|c|}
\hline \multirow[b]{3}{*}{ Variable } & \multirow{2}{*}{\multicolumn{3}{|c|}{ 3-way ANOVA }} & \multicolumn{6}{|c|}{$b L E D$} & \multicolumn{6}{|c|}{$N A L$} \\
\hline & & & & \multicolumn{2}{|c|}{ AO } & \multicolumn{2}{|c|}{$A 1$} & \multicolumn{2}{|c|}{$A 2$} & \multicolumn{2}{|c|}{$A O$} & \multicolumn{2}{|c|}{$A 1$} & \multicolumn{2}{|c|}{$A 2$} \\
\hline & $L$ & $H$ & $S$ & Day & Night & Day & Night & Day & Night & Day & Night & Day & Night & Day & Night \\
\hline $\mathrm{SpO}_{2}(\%)$ & NS & & $d$ & $99 \pm 1$ & $99 \pm 1$ & $84 \pm 3^{e}$ & $80 \pm 7^{e g}$ & $81 \pm 6^{e f}$ & $76 \pm 6^{e f g}$ & $98 \pm 3$ & $99 \pm 1$ & $84 \pm 6^{e}$ & $79 \pm 6^{e g}$ & $81 \pm 5^{e}$ & $80 \pm 4^{e g}$ \\
\hline HR (bpm) & NS & $a b$ & $d$ & $78 \pm 11$ & $84 \pm 11^{g}$ & $84 \pm 11^{e}$ & $93 \pm 10^{e g}$ & $85 \pm 10^{e}$ & $96 \pm 8^{e f g}$ & $75 \pm 10$ & $87 \pm 6^{g}$ & $80 \pm 10^{e}$ & $91 \pm 8^{e g}$ & $86 \pm 13^{e f}$ & $95 \pm 9^{e g}$ \\
\hline $\mathrm{SP}(\mathrm{mm} \mathrm{Hg})$ & NS & $a b$ & d & $115 \pm 11$ & $120 \pm 15^{g}$ & $106 \pm 7^{e}$ & $116 \pm 12^{e g}$ & $106 \pm 9^{e}$ & $110 \pm 11^{e f}$ & $115 \pm 11$ & $119 \pm 14^{e}$ & $108 \pm 7^{e}$ & $113 \pm 12^{e g}$ & $111 \pm 20$ & $110 \pm 11^{e}$ \\
\hline $\begin{array}{l}\text { DP }(m m ~ H g) \\
\text { HRV }\end{array}$ & NS & NS & $d$ & $71 \pm 7$ & $78 \pm 10^{g}$ & $68 \pm 7$ & $75 \pm 14^{g}$ & $69 \pm 9$ & $76 \pm 13^{g}$ & $70 \pm 11$ & $76 \pm 9^{g}$ & $66 \pm 6$ & $77 \pm 14^{g}$ & $69 \pm 7$ & $80 \pm 15^{g}$ \\
\hline SDNN & NS & $a b$ & NS & $57 \pm 22$ & $65=$ & $52 \pm 21$ & $67 \pm 32$ & $51 \pm 21$ & $48 \pm 21^{e f}$ & $62 \pm 22$ & $71 \pm 20$ & $64 \pm 31$ & $81 \pm 61$ & $55 \pm 24$ & $46 \pm 25^{e f}$ \\
\hline RMSSD (ms) & NS & NS & $d$ & $52 \pm 30$ & $42 \pm 17$ & $41 \pm 26$ & $35 \pm 19$ & $41 \pm 25$ & $25 \pm 12^{g}$ & $54 \pm 27$ & $43 \pm 16$ & $51 \pm 32$ & $48 \pm 39$ & $46 \pm 23$ & $25 \pm 19^{g}$ \\
\hline pNN50 (\%) & NS & $a b$ & $d$ & $28 \pm 19$ & $20 \pm 16$ & $14 \pm 14^{e}$ & $13 \pm 14$ & $13 \pm 10^{e}$ & $7 \pm 9^{e f}$ & $26 \pm 21$ & $22 \pm 13$ & $20 \pm 18$ & $13 \pm 16^{e}$ & $20 \pm 16$ & $7 \pm 13^{e f g}$ \\
\hline VLF (\%) & NS & NS & $d$ & $18 \pm 10$ & $35 \pm 19^{g}$ & $26 \pm 13$ & $44 \pm 16^{g}$ & $20 \pm 13$ & $42 \pm 13^{g}$ & $23 \pm 13$ & $46 \pm 17^{g}$ & $25 \pm 13$ & $47 \pm 22^{g}$ & $19 \pm 10$ & $46 \pm 16^{g}$ \\
\hline LF $(\%)$ & NS & $b$ & $\mathrm{~N}$ & $39 \pm 9$ & $38 \pm 15$ & $43 \pm 11$ & $36 \pm 13$ & $53 \pm 16^{e}$ & $42 \pm 11$ & $36 \pm 14$ & $33 \pm 10$ & $39 \pm 14$ & $31 \pm 12$ & $46 \pm 12^{e}$ & $40 \pm 12$ \\
\hline $\mathrm{HF}(\%)$ & NS & $b$ & 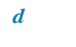 & $43 \pm 15$ & $27 \pm 14^{g}$ & $32 \pm 20^{e}$ & $20 \pm 7^{g}$ & $28 \pm 14^{e}$ & $16 \pm 6^{g}$ & $42 \pm 22$ & $21 \pm 11^{g}$ & $36 \pm 22$ & $22 \pm 14$ & $36 \pm 16$ & $14 \pm 6^{g}$ \\
\hline $\mathrm{LF} / \mathrm{HF}$ & NS & $b$ & NS & $1.3 \pm 1.7$ & $2.1 \pm 1.8$ & $2.5 \pm 2.6$ & $2.2 \pm 1.5$ & $2.9 \pm 3.0$ & $3.1 \pm 1.6^{e}$ & $1.5 \pm 1.6$ & $1.9 \pm 0.9$ & $2.1 \pm 2.2$ & $1.8 \pm 1.2$ & $2.2 \pm 1.9$ & $3.8 \pm 2.9^{e}$ \\
\hline
\end{tabular}

All data are mean \pm SD. Multiple comparisons were performed by a 3-way analysis of variance (3-way ANOVA) with factors L (light, with levels bLED and NAL), H (hypoxia, with levels A0, $\mathrm{A} 1$, and A2), and S (schedule, with levels day and night). Holm-Sidak and paired $t$ tests were used in post-hoc analysis for pairwise comparisons within levels.

bLED, blue LED; NAL, normal artificial light; A0, at sea level; A1, 15 minutes after ascent to simulated altitude of $4000 \mathrm{~m}$; A2, 3 hours of simulated altitude of $4000 \mathrm{~m}$; Spo , arterial saturation; NS, not significant; HR, heart rate; SP, systolic blood pressure; DP, diastolic blood pressure; HRV, heart rate variability; SDNN, standard deviation of R-R intervals; RMSSD, square differences of successive R-R intervals; pNN50, differences of successive intervals greater than $50 \mathrm{~ms}$; VLF, very low frequency; LF, low frequency; HF, high frequency.

${ }^{a} \mathrm{~A} 0$ vs $\mathrm{A} 1, P<.05,3$-way ANOVA.

${ }^{b} \mathrm{~A} 0$ vs $\mathrm{A} 2, P<.05,3$-way ANOVA.

${ }^{c} \mathrm{~A} 1$ vs $\mathrm{A} 2, P<.05,3$-way ANOVA.

${ }^{d}$ day vs night, $P<.05,3$-way ANOVA.

$e^{e}$ vs A $0, P<.05$, Holm-Sidak and paired $t$ tests.

${ }^{f}$ vs A1, $P<.05$, Holm-Sidak and paired $t$ tests.

${ }^{g}$ vs day, $P<.05$, Holm-Sidak and paired $t$ tests. 
Table 4. Cognitive parameters in men and women exposed to 2 kinds of light under normobaric normoxic and hypobaric hypoxic conditions

\begin{tabular}{|c|c|c|c|c|c|c|c|c|c|c|c|c|c|c|c|c|}
\hline \multirow[b]{3}{*}{ Variable } & \multirow[b]{3}{*}{$\operatorname{Sex}$} & & & & \multicolumn{6}{|c|}{$b L E D$} & \multicolumn{6}{|c|}{$N A L$} \\
\hline & & \multicolumn{3}{|c|}{ 3-way ANOVA } & \multicolumn{2}{|c|}{$A O$} & \multicolumn{2}{|c|}{$A 1$} & \multicolumn{2}{|c|}{$A 2$} & \multicolumn{2}{|c|}{$A O$} & \multicolumn{2}{|c|}{$A 1$} & \multicolumn{2}{|c|}{$A 2$} \\
\hline & & $L$ & $H$ & $S$ & Day & Night & Day & Night & Day & Night & Day & Night & Day & Night & Day & Night \\
\hline \multicolumn{17}{|l|}{$\mathrm{d} 2$ test } \\
\hline \multirow[t]{2}{*}{ TOT } & Men & NS & $b$ & $d$ & $546 \pm 78$ & $475 \pm 107^{g}$ & $581 \pm 69^{e}$ & $544 \pm 85^{e}$ & $573 \pm 82^{e}$ & $530 \pm 93^{e}$ & $530 \pm 85$ & $501 \pm 120$ & $572 \pm 77^{e}$ & $541 \pm 77$ & $562 \pm 90^{e}$ & $529 \pm 87$ \\
\hline & Women & NS & $b c$ & $d$ & $555 \pm 70$ & $482 \pm 125^{g}$ & $609 \pm 42^{e}$ & $546 \pm 92^{e g}$ & $598 \pm 44^{e}$ & $531 \pm 95^{e g}$ & $577 \pm 72$ & $462 \pm 127^{g}$ & $618 \pm 36^{e}$ & $520 \pm 111^{e g}$ & $613 \pm 35^{e}$ & $487 \pm 114^{f g}$ \\
\hline \multirow[t]{2}{*}{$\mathrm{CON}$} & Men & NS & $b$ & NS & $230 \pm 45$ & $217 \pm 42$ & $250 \pm 39^{e}$ & $246 \pm 36^{e}$ & $244 \pm 40^{e}$ & $241 \pm 37^{e}$ & $219 \pm 47$ & $227 \pm 50$ & $243 \pm 46^{e}$ & $244 \pm 34$ & $243 \pm 48^{e}$ & $241 \pm 35$ \\
\hline & Women & NS & $b c$ & $d$ & $231 \pm 47$ & $219 \pm 48$ & $266 \pm 31^{e}$ & $248 \pm 37^{e}$ & $256 \pm 35^{e}$ & $243 \pm 35^{e}$ & $249 \pm 46$ & $206 \pm 47^{g}$ & $271 \pm 26^{e}$ & $234 \pm 41^{g}$ & $269 \pm 24^{e}$ & $223 \pm 39^{g}$ \\
\hline \multicolumn{17}{|l|}{$\mathrm{AB}$} \\
\hline \multirow[t]{2}{*}{$\mathrm{RT}(\mathrm{s})$} & Men & $a$ & NS & $d$ & $3.5 \pm 0.3$ & $3.1 \pm 0.3^{g}$ & $3.4 \pm 0.2$ & $3.0 \pm 0.3^{g}$ & $3.3 \pm 0.3$ & $2.9 \pm 0.3^{g}$ & $3.4 \pm 0.3$ & $2.9 \pm 0.2^{g}$ & $3.2 \pm 0.3$ & $2.8 \pm 0.1^{g}$ & $3.3 \pm 0.3$ & $2.8 \pm 0.1^{g}$ \\
\hline & Women & NS & $b c$ & $d$ & $3.4 \pm 0.3$ & $3.0 \pm 0.4^{g}$ & $3.2 \pm 0.4^{e}$ & $2.9 \pm 0.4^{e g}$ & $3.2 \pm 0.3^{e}$ & $2.9 \pm 0.4^{g}$ & $3.6 \pm 0.3$ & $3.0 \pm 0.2^{g}$ & $3.5 \pm 0.3^{e}$ & $2.8 \pm 0.3^{e g}$ & $3.3 \pm 0.3^{e}$ & $2.8 \pm 0.2^{e g}$ \\
\hline \multirow[t]{2}{*}{ RA (\%) } & Men & NS & NS & NS & $95 \pm 2$ & $95 \pm 4$ & $95 \pm 4$ & $93 \pm 6$ & $94 \pm 5$ & $93 \pm 5$ & $95 \pm 4$ & $95 \pm 8$ & $97 \pm 4$ & $94 \pm 4$ & $96 \pm 4$ & $95 \pm 5$ \\
\hline & Women & NS & NS & d & $91 \pm 14$ & $84 \pm 24$ & $92 \pm 11$ & $85 \pm 22$ & $94 \pm 12$ & $86 \pm 21^{g}$ & $94 \pm 9$ & $88 \pm 12$ & $93 \pm 9$ & $87 \pm 14$ & $95 \pm 9$ & $88 \pm 15$ \\
\hline
\end{tabular}

All data are mean \pm SD. Multiple comparisons were performed by a 3-way analysis of variance (3-way ANOVA) with factors L (light, with levels bLED and NAL), H (hypoxia, with levels A0, $\mathrm{A} 1$, and $\mathrm{A} 2$ ), and $\mathrm{S}$ (schedule, with levels day and night). Holm-Sidak and paired $t$ tests were used in post-hoc analysis for pairwise comparisons within levels.

bLED, blue LED; NAL, normal artificial light; A0, at sea level; A1, 15 minutes after ascent to simulated altitude of $4000 \mathrm{~m}$; A2, 3 hours of simulated altitude of 4000 m; NS, not significant; TOT, total effectiveness; $\mathrm{CON}$, concentration index; $\mathrm{AB}$, attentional blink; RT, reaction time; RA, right answers.

${ }^{a}$ bLED vs NAL, $P<.05,3$-way ANOVA.

${ }^{b} \mathrm{~A} 0$ vs A1, $P<.05$, 3-way ANOVA.

A0 vs A2, $P<.05,3$-way ANOVA.

${ }^{d}$ day vs night, $P<.05$, 3-way ANOVA.

${ }^{e}$ vs A0, $P<.05$, Holm-Sidak and paired $t$ tests.

${ }^{f}$ vs A1, $P<.05$, Holm-Sidak and paired $t$ tests.

${ }^{g}$ vs day, $P<.05$, Holm-Sidak and paired $t$ tests. 
showed opposite trends: VLF increased at night whereas HF decreased. The effects of hypoxia on HRV were especially notable after 3 hours of hypoxia (A2). Significant reductions were observed in both men and women in pNN50 and HF, along with significant increases in LF. Moreover, the LF/HF ratio presented higher values at A2 in both men and women, although they were only statistically significant in women. Regarding the effect of bLED on HRV, the only significant difference with respect to NAL was a reduction in SDNN in men at night after 3 hours of hypobaric exposure (A2), and not significantly lower values in the time domain parameters (RMSSD and pNN50) also at night and A2.

\section{COGNITIVE PARAMETERS}

Although bLED did not show any statistically significant differences in the parameters derived from the $\mathrm{d} 2$ test (TOT and CON), there was a slight tendency for both parameters to increase at day (Table 4). The parameters of this test showed significantly higher values in women (both in normoxia and hypoxia and in both lighting conditions) in the daytime. Surprisingly, TOT and CON increased significantly under hypoxic conditions. RT in the $A B$ test was significantly higher in both men and women in the daytime and presented a significant increase in men as a result of bLED illumination; posthoc multiple comparison test detected global significant differences for light factor.

\section{Discussion}

The protocol of hypobaric hypoxia (short-term artificial hypoxia conditions in a hypobaric chamber) was designed to avoid the risk of severe AMS symptoms in the subjects. Moreover, this approach allows us to isolate hypoxia effects from other environmental factors (such as cold, dry atmosphere, or strong wind) and also to overcome the difficulties of performing a similar study under real field conditions (eg, work schedule, additional stress, and time availability). Altitude hypoxia is an environmental factor that strongly affects human body functions, eliciting a series of compensatory physiological responses, such as tachycardia and hyperventilation (for an extensive review, see West et $\mathrm{al}^{1}$ ). These cardiorespiratory responses are driven by general and sustained sympathetic activation that favors the occurrence of fatigue. ${ }^{37} \mathrm{~A}$ total of $41.7 \%$ of Chilean mineworkers declared physical/physiological or psychological/cognitive fatigue at geographical altitude, which is a positive predictor for AMS. ${ }^{38}$ The role of bLED as a possible fatigue regulator has been studied and can be considered as well established at sea level. ${ }^{18,31,39,40}$ However, the feasibility of applying it at high altitude has not been verified, and the possible effects of bLED illumination on shift work at altitude are not known.

\section{ARTERIAL OXYGEN SATURATION}

Inspired $\mathrm{PO}_{2}$ decreases with barometric pressure; at a simulated altitude of $4000 \mathrm{~m}$, it is approximately $90 \mathrm{~mm}$ $\mathrm{Hg}$, which corresponds to $60 \%$ of the inspired $\mathrm{Po}_{2}$ at sea level. ${ }^{41}$ This fall in $\mathrm{PO}_{2}$ is responsible for the well-known reduction in $\mathrm{SpO}_{2}$ provoked by acute hypobaric conditions. As expected, we found a reduction of $\mathrm{SpO}_{2}$ levels in hypobaric conditions (Tables 2 and 3) in agreement, for example, with the diurnal values $(81 \%$ $85 \%$ ) found by Shen et $\mathrm{al}^{42}$ in a recent field study at $4000 \mathrm{~m}$.

One of the interesting findings in the present study is the circadian effect reflected in the $\mathrm{Spo}_{2}$ levels recorded at night under hypobaric conditions (A1 and A2): There was a significant decrease, $4 \%$ to $9 \%$, in absolute $\mathrm{SpO}_{2}$ values. It is important to note that all subjects were awake and had no apparent signs of periodic breathing. This effect was not apparent under normoxia (A0), when the values fell in the normal range of saturation under sea-level conditions $(98 \%-100 \%)$ both during the day and at night.

A circadian rhythm in peak expiratory flow has been reported, an indicator of bronchial diameter. Reinberg et $\mathrm{al}^{43}$ reported a $10 \%$ reduction in peak expiratory flow between 2300 hours and 0700 hours. Adamcyk et $\mathrm{al}^{44}$ also reported significant reductions in several respiratory parameters such as tidal volume, minute ventilation, and mean inspiratory flow at night. This circadian rhythm could play a role in explaining the more marked reductions in $\mathrm{Spo}_{2}$ at night during acute hypoxia. An alteration in oxygen-conducting pathways from atmosphere to blood, such as a reduction in bronchial diameter, could have a greater effect during acute hypoxia, when the fall in environmental $\mathrm{PO}_{2}$ compromises oxygen homeostasis, than in normoxia, thereby explaining the differences found between night and day in hypoxia but not in normoxia.

\section{BLOOD PRESSURE}

Our results show a circadian rhythm in systolic and diastolic blood pressure in women, with significant increases in almost all situations at night. This agrees with the results reported by Hermida et $a 1,{ }^{45}$ who found 2 peaks of arterial pressure values at 4 hours and 12 hours (before night rest) after waking, but is in clear contrast with the circadian rhythm described by Cagnacci et al, ${ }^{46}$ 
who reported a decline in blood pressure at night that is enhanced by the prolonged administration of melatonin.

In men, we also obtained higher values at night, but they were not significant, introducing a source of variation between men and women in the present study that contrasts with the previously reported circadian decline in young men. ${ }^{47}$ Regarding the effect of acute hypoxia on systolic and diastolic arterial pressure, our results contrast with those obtained at similar altitudes by other authors. Wolfel et $\mathrm{al}^{48}$ reported a rise in systemic blood pressure in men after 2 days at $4300 \mathrm{~m}$ and attributed the increase to sympathetic activity. Rapid ascents to $4559 \mathrm{~m}$ (in $<28$ hours or after being airlifted by helicopter) have also been reported to result in significant increases both in systolic and diastolic blood pressures. ${ }^{49,50}$ However, it should be noted that all of these studies were only of men, with a wide range of ages, who were exposed under natural hypoxic conditions.

In contrast, in a study performed in a hypobaric chamber, at a simulated altitude of $4800 \mathrm{~m}$ and with the same short hypoxic exposure time of 3 hours as that used in the present study, Loshbaugh et $\mathrm{al}^{51}$ reported no significant increases in mean blood pressure (calculated from systolic and diastolic measurements) in 6 men and 3 women with the same mean age as the subjects participating in our study. Thus, a possible explanation for the contrasting results obtained in our hypobaric chamber could be both the short time of hypoxic exposure ( 3 hours) and the fact that under field exposure there is a wide range of environmental variables (eg, temperature) that cannot be controlled and could play a role in arterial blood pressure changes.

\section{HEART RATE}

The protocol of acute hypobaric hypoxia followed in this research showed significant increases in heart rate in all cases, ranging from $7 \%$ to $13 \%$. These higher heart rates were observed immediately after reaching the simulated altitude of $4000 \mathrm{~m}$ (A1) and lasted for the entire 3 hours of hypoxia (A2). This observation has been repeatedly confirmed, both in the field and in simulated altitude (see, for example, Naeije ${ }^{52}$ ), and leads to an increase in cardiac output and therefore higher oxygen delivery to tissues. Our results also show a circadian rhythm in heart rate, with significant increases at night that range from $8 \%$ to $18 \%$ when compared with daytime. The circadian rhythm of heart rate has been extensively studied, and clear diurnal and nocturnal variations have been reported and attributed to increased sympathetic activity during the day. ${ }^{53}$
Our results in normoxia (A0) are in close agreement with a study by Hermida et $\mathrm{al}^{45}$ in white men and women of the same age, with increases of between $8 \%$ and $16 \%$ at night. Moreover, our results show that the circadian rhythms are maintained in hypobaric hypoxia, both immediately after reaching $4000 \mathrm{~m}$ (A1, with increases of $11 \%-14 \%$ at night) and after 3 hours of exposure (A2, with increases of $10 \%-18 \%$ at night). Finally, we must conclude that there is no effect of bLED on heart rate, either when compared with NAL or regarding circadian rhythm.

\section{HEART RATE VARIABILITY}

As previously described, acute altitude exposure induced a marked decrease in $\mathrm{HRV} \cdot{ }^{54-56}$ We found some significant circadian changes in HRV that could reflect variations in autonomous nervous system activity. Especially in women, a significantly lower beat-to-beat HRV (ie, a reduction in parameters such as RMSSD and pNN50) together with a significant decrease in HF at night may indicate reduced vagal activity that matches the significant increases in heart rate discussed above. We also found a significant reduction in VLF at night in both men and women.

Although the physiological mechanisms underlying VLF oscillations are still unclear, fluctuations of thermoregulatory activity and parasympathetic outflow have been proposed as potential factors ${ }^{57}$ and could explain these results. Hypobaric hypoxia had significant effects on HRV, especially after 3 hours of exposure (A2): we found decreases in parameters reflecting parasympathetic modulation (pNN50 and HF) and increases in LF and the LF/HF ratio. All these results are indicative of activation of the sympathetic tone and a vagal withdrawal response, in agreement with previous work on acute hypoxia at rest. ${ }^{58-61}$ Apart from a significant reduction in SDNN in men at night after 3 hours of hypobaric exposure, in the present study we did not find any clear-cut trend in the effect of bLED illumination on HRV. These findings suggest a negligible effect of blue light on HRV, at least in the first 3 hours of exposure.

\section{COGNITIVE PERFORMANCE}

Beside the effects of hypobaric hypoxia on physiological parameters, a variety of effects on neuropsychological functions have also been reported that have mostly been attributed to cerebral hypoxia and the resulting functional and structural cerebral alterations. Some studies have demonstrated short-term visual and spatial memory deterioration when ascending over $2500 \mathrm{~m},{ }^{62,63}$ with particularly notable effects over $4500 \mathrm{~m} .{ }^{64}$ Those studies also found a reduction in the speed and precision of 
motor capacity in comparison to sea level conditions. Language disorders, such as aphasias and deficiencies in comprehension, have also been reported in mountaineers, which affect them in an altitude-dependent pattern. ${ }^{65,66} \mathrm{~A}$ decrease in reaction time, psychomotor ability, and mental efficiency is also a common finding in altitude-exposed subjects in both experiments performed in hypobaric chambers $^{67,68}$ and during mountain expeditions. ${ }^{69}$ Moreover, a rapid ascent to altitude is associated with decreased function and cognitive performance in subjects trained in specific jobs, showing deterioration in accuracy tests and a decrease in execution speed caused by hypoxia. $^{70}$

On the other hand, it has been established that the circadian cycle is sensitive to blue light exposure. ${ }^{17}$ Blue light increases alertness of subjects, in association with a higher subjective feeling of vigil and low levels of fatigue at night, ${ }^{31,40,71}$ although there is a significant decrease in subjective sleepiness during 460-nm light exposure compared with 550-nm light exposure and no light, starting 30 minutes after lights on. ${ }^{72}$ The use of enriched blue light has been generalized in places of work, such as in the cabins of heavy vehicles or aircrafts, ${ }^{31}$ because of a demonstrated effect of reducing fatigue in workers. However, to our knowledge, no previous studies assessed the potential positive effects of blue light under acute hypobaric hypoxia. It is important to note that hypoxic conditions greatly alter most physiological control mechanisms; for this reason, it is necessary to confirm the putative positive effects of blue light, which have been well established at sea level, under these environmental conditions.

Although our results are statistically insignificant, a tendency to solve the $\mathrm{d} 2$ attention test with better cognitive performance was detected in some cases. Thus, in men, the overall TOT and CON tended to be higher $(2 \%-5 \%)$ when the test was executed under bLED in the day (Table 4). In women, bLED resulted in a tendency to improve cognitive performance with increases in TOT and $\mathrm{CON}$ from $4 \%$ to $8 \%$; however, in this case, this was true when the $\mathrm{d} 2$ test was performed at night (Table 4). Moreover, in women there is a significant difference (both in normoxia and hypoxia and in both lighting conditions) in cognitive performance: they perform better in the $\mathrm{d} 2$ test during the daytime than at night.

A surprising finding of our study is that for both lightings, TOT and CON were significantly improved under hypoxic conditions. We believe that this finding arises from an important limitation of the study: a probable training effect derived from the repeated, close in time executions of the $\mathrm{d} 2$ test. This effect would be the reason for consistently better performance in subsequent tests compared with the first one. This is also an important methodological limitation to be considered for d2 test field applications (ie, altitude workers).

Regarding the results of the $\mathrm{AB}$ test, significantly lower RT scores were found at night in both men and women. This finding agrees with studies that demonstrate that the circadian fluctuations of serial-visual performance in a vigilance task have an interval for maximum speed execution between 1900 hours and 2100 hours. $^{73}$ However, in women the significant reduction in RT found at night is accompanied by a loss of effectiveness (lower RA) in comparison with daytime. Contrasting the trend observed in parameters from the $\mathrm{d} 2$ test, RT significantly increased in men under bLED illumination, indicating another difference between the sexes and questioning the usefulness of bLED in reaction tasks.

\section{Conclusions}

In conclusion, we observed significant circadian rhythm differences in physiological parameters such as blood pressure, heart rate, and HRV, which are maintained in acute hypoxia after 3 hours of exposure. Moreover, a circadian effect on $\mathrm{SpO}_{2}$ under acute hypoxia conditions has been observed, with significant decreases at night. Sex differences were also evident, with different behavior in the physiological and cognitive parameters after hypoxia. Some tendencies toward better cognitive performance from the $\mathrm{d} 2$ attention test under bLED illumination were seen, although when considered with the physiological parameters and reaction time, there was no conclusive favorable effect of bLED on fatigue suppressive effects after 3 hours of acute hypobaric hypoxia. It remains to be seen whether exposure times to bLED longer than 3 hours under hypobaric hypoxic conditions would induce favorable effects against fatigue. These conclusions are limited by the present study design, mainly the short-term hypoxia exposure to avoid the risk of severe AMS in the subjects owing to the lack of acclimatization.

\section{Acknowledgments}

The authors are grateful to all the students of the UB/ UPC MSc in Biomedical Engineering and the UB BSc in Biomedical Sciences who kindly agreed to participate in the study. This study was funded by grants A/025717/09 and A/030280/10 from the Spanish Agencia Española de Cooperación Internacional y Desarrollo (AECID) and the Chilean FONDECYT project no. 1100161. We are also grateful to Prof JA Aznar from the Department of Basic Psychology at the University of Barcelona for providing us with the software for the attentional blink test. The authors do not report any potential conflicts of interest. 


\section{References}

1. West JB, Milledge JS, Schoene RB, Luks A, eds. High Altitude Medicine and Physiology. 5th ed. Boca Raton, FL: CRC Press/Taylor \& Francis Group; 2013.

2. Mazzeo RS. Altitude, exercise and immune function. Exerc Immunol Rev. 2005;11:6-16.

3. Wilber RL. Application of altitude/hypoxic training by elite athletes. Med Sci Sports Exerc. 2007;39:1610-1624.

4. Zhang H, Lin J, Sun Y, et al. Compromised white matter microstructural integrity after mountain climbing: evidence from diffusion tensor imaging. High Alt Med Biol. 2012; 13:118-125.

5. Zhang G, Zhou S-M, Yuan C, Tian H-J, Li P, Gao Y-Q. The effects of short-term and long-term exposure to a high altitude hypoxic environment on neurobehavioral function. High Alt Med Biol. 2013;14. 338-41.

6. Richalet JP, Donoso MV, Jiménez D, et al. Chilean miners commuting from sea level to $4500 \mathrm{~m}$ : a prospective study. High Alt Med Biol. 2002;3:159-166.

7. Gerard AB, McElroy MK, Taylor MJ, et al. Six percent oxygen enrichment of room air at simulated 5,000 m altitude improves neuropsychological function. High Alt Med Biol. 2000;1:51-61.

8. Behn C, Dinamarca GA, De Gregorio NF, et al. Agerelated arrhythmogenesis on ascent and descent: "autonomic conflicts" on hypoxia/reoxygenation at high altitude? High Alt Med Biol. 2014;15:356-363.

9. Bärtsch P, Saltin B. General introduction to altitude adaptation and mountain sickness. Scand J Med Sci Sports. 2008;18(suppl 1):1-10.

10. Luks AM, McIntosh SE, Grissom CK, et al. Wilderness Medical Society. Wilderness Medical Society consensus guidelines for the prevention and treatment of acute altitude illness. Wilderness Environ Med. 2010;21:146-155.

11. Muza SR, Beidleman BA, Fulco CS. Altitude preexposure recommendations for inducing acclimatization. High Alt Med Biol. 2010;11:87-92.

12. Richalet JP, Bittel J, Herry JP, et al. Use of a hypobaric chamber for pre-acclimatization before climbing Mount Everest. Int J Sports Med. 1992;13(suppl 1):S216-S220.

13. Böcker M, Vogy J, Nolle-Gösser T. Scientific approach for optimising performance, health and safety in high-altitude observatories. Messenger (Los Angel). 2008;133:49-52.

14. West JB. Oxygen enrichment of room air to relieve the hypoxia of high altitude. Respir Physiol. 1995;99:225-232.

15. West JB. Commuting to high altitude: value of oxygen enrichment of room air. High Alt Med Biol. 2002;3:223-235.

16. West JB. Improving oxygenation at high altitude: acclimatization and $\mathrm{O}_{2}$ enrichment. High Alt Med Biol. 2003;4: 389-398.

17. Brainard GC, Hanifin JP, Greeson JM, et al. Action spectrum for melatonin regulation in humans: evidence for a novel circadian photoreceptor. J Neurosci. 2001;21:6405-6412.

18. Figueiro MG, Bierman A, Plitnick B, Rea MS. Preliminary evidence that both blue and red light can induce alertness at night. BMC Neurosci. 2009;10:105.
19. Mainster MA, Turner PL. Blue light's benefits vs blueblocking intraocular lens chromophores. Graefes Arch Clin Exp Ophthalmol. 2012;250:1245-1246.

20. Münch M, Léon L, Crippa SV, Kawasaki A. Circadian and wake-dependent effects on the pupil light reflex in response to narrow-bandwidth light pulses. Invest Ophthalmol Vis Sci. 2012;53:4546-4555.

21. Moore RY, Lenn NJ. A retinohypothalamic projection in the rat. J Comp Neurol. 1972;146:1-14.

22. Sekaran S, Foster RG, Lucas RJ, Hankins MW. Calcium imaging reveals a network of intrinsically light-sensitive inner-retinal neurons. Curr Biol. 2003;13:1290-1298.

23. Moore RY. Organization and function of a central nervous system circadian oscillator: the suprachiasmatic hypothalamic nucleus. Fed Proc. 1983;42:2783-2789.

24. Argamaso SM, Froehlich AC, McCall MA, Nevo E, Provencio I, Foster RG. Photopigments and circadian systems of vertebrates. Biophys Chem. 1995;56:3-11.

25. Macchi MM, Bruce JN. Human pineal physiology and functional significance of melatonin. Front Neuroendocrinol. 2004;25:177-195.

26. Viola AU, James LM, Schlangen LJM, Dijk DJ. Blueenriched white light in the workplace improves selfreported alertness, performance and sleep quality. Scand J Work Environ Health. 2008;34:297-306.

27. Chen Y, Tjong Y-W, Ip S-F, Tipoe GL, Fung M-L. Melatonin enhances the hypoxic response of rat carotid body chemoreceptor. J Pineal Res. 2005;38. 157-63.

28. Tekavcic-Pompe M, Tekavcic I. Color vision in the tritan axis is predominantly affected at high altitude. High Alt Med Biol. 2008;9:38-42.

29. Vandewalle G, Gais S, Schabus M, et al. Wavelengthdependent modulation of brain responses to a working memory task by daytime light exposure. Cereb Cortex. 2007; 17:2788-2795.

30. Vandewalle G, Maquet P, Dijk D-J. Light as a modulator of cognitive brain function. Trends Cogn Sci. 2009; 13:429-438.

31. Phipps-Nelson J, Redman JR, Schlangen LJM, Rajaratnam SMW. Blue light exposure reduces objective measures of sleepiness during prolonged nighttime performance testing. Chronobiol Int. 2009;26:891-912.

32. Brickenkamp R. The d2 Test of Attention. Göttingen, Germany: Hogrefe; 1962.

33. Raymond JE, Shapiro KL, Arnell KM. Temporary suppression of visual processing in an RSVP task: an attentional blink? J Exp Psychol Hum Percept Perform. 1992;18:849-860.

34. Tarvainen MP, Niskanen J-P, Lipponen JA, Ranta-Aho PO, Karjalainen PA. Kubios HRV—heart rate variability analysis software. Comput Methods Programs Biomed. 2014; 113:210-220.

35. Seisdedos N. d2, Test de Atención. (Spanish adaptation.). Madrid, Spain: TEA Ediciones; 2002.

36. Chun MM, Potter MC. A two-stage model for multiple target detection in rapid serial visual presentation. $J$ Exp Psychol Hum Percept Perform. 1995;21:109-127. 
37. Tran $\mathrm{Y}$, Wijesuriya $\mathrm{N}$, Tarvainen $\mathrm{M}$, Karjalainen $\mathrm{P}$, Craig A. The relationship between spectral changes in heart rate variability and fatigue. J Psychophysiol. 2009; 23:143-151.

38. Vera A, Carrasco C, Vanegas J, Contreras G. Fatiga física y fatiga cognitiva en trabajadores de la minería que laboran en condiciones de altitud geográfica. Relación con el mal agudo de montaña. Cienc Trab. 2008;29:90-94.

39. Bennett S, Alpert M, Kubulins V, Hansler RL. Use of modified spectacles and light bulbs to block blue light at night may prevent postpartum depression. Med Hypotheses. 2009;73:251-253.

40. Taillard J, Capelli A, Sagaspe P, Anund A, Akerstedt T, Philip P. In-car nocturnal blue light exposure improves motorway driving: a randomized controlled trial. PLOS One. 2012;7:e46750.

41. West JB. Prediction of barometric pressures at high altitude with the use of model atmospheres. J Appl Physiol. 1996;81:1850-1854.

42. Shen G, Xie K, Yan Y, et al. The role of oxygen-increased respirator in humans ascending to high altitude. Biomed Eng Online. 2012;11:49-56.

43. Reinberg A, Guillet P, Gervais P, Ghata J, Vignaud D, Abulker C. One month chronocorticotherapy (Dutimelan 8 15 mite). Control of the asthmatic condition without adrenal suppression and circadian rhythm alteration. Chronobiologia. 1977;4:295-312.

44. Adamczyk W, Tafil-Klawe M, Siekierka M, Zlomanczuk P, Weber P, Klawe JJ. Daily pattern of breathing in healthy young men. J Physiol Pharmacol. 2008;59(suppl 6):115-122.

45. Hermida RC, Ayala DE, Fernández JR, Mojón A, Alonso I, Calvo C. Modeling the circadian variability of ambulatorily monitored blood pressure by multiple-component analysis. Chronobiol Int. 2002;19:461-481.

46. Cagnacci A, Cannoletta M, Renzi A, Baldassari F, Arangino S, Volpe A. Prolonged melatonin administration decreases nocturnal blood pressure in women. Am J Hypertens. 2005;18(12 Pt 1):1614-1618.

47. Degaute JP, van de Borne P, Linkowski P, Van Cauter E. Quantitative analysis of the 24-hour blood pressure and heart rate patterns in young men. Hypertension. 1991;18:199-210.

48. Wolfel EE, Selland MA, Mazzeo RS, Reeves JT. Systemic hypertension at $4,300 \mathrm{~m}$ is related to sympathoadrenal activity. J Appl Physiol. 1994;76:1643-1650.

49. Kanstrup IL, Poulsen TD, Hansen JM, et al. Blood pressure and plasma catecholamines in acute and prolonged hypoxia: effects of local hypothermia. J Appl Physiol. 1999;87:2053-2058.

50. Parati G, Revera M, Giuliano A, et al. Effects of acetazolamide on central blood pressure, peripheral blood pressure, and arterial distensibility at acute high altitude exposure. Eur Heart J. 2013;34:759-766.

51. Loshbaugh JE, Loeppky JA, Greene ER. Effects of acute hypobaric hypoxia on resting and postprandial superior mesenteric artery blood flow. High Alt Med Biol. 2006;7: 47-53.
52. Naeije R. Physiological adaptation of the cardiovascular system to high altitude. Prog Cardiovasc Dis. 2010;52: 456-466.

53. Guo YF, Stein PK. Circadian rhythm in the cardiovascular system: chronocardiology. Am Heart J. 2003;145:779-786.

54. Cornolo J, Mollard P, Brugniaux JV, Robach P, Richalet JP. Autonomic control of the cardiovascular system during acclimatization to high altitude: effects of sildenafil. $J$ Appl Physiol. 2004;97:935-940.

55. Schmitt L, Fouillot JP, Millet GP, et al. Altitude, heart rate variability and aerobic capacities. Int J Sports Med. 2008; 29:300-306.

56. Basualto-Alarcón C, Rodas G, Galilea PA, et al. Cardiorespiratory parameters during submaximal exercise under acute exposure to normobaric and hypobaric hypoxia. Apunt Med Esport. 2012;47:65-72.

57. Taylor JA, Carr DL, Myers CW, Eckberg DL. Mechanisms underlying very-low-frequency RR-interval oscillations in humans. Circulation. 1998;98:547-555.

58. Buchheit M, Richard R, Doutreleau S, Lonsdorfer-Wolf E, Brandenberger G, Simon C. Effect of acute hypoxia on heart rate variability at rest and during exercise. Int $J$ Sports Med. 2004;25:264-269.

59. Fukuda-Matsuda E, Yamada M, Tanobe K, Saito S. Peripheral circulation monitored by surface temperature and autonomic nervous function in hypobaric hypoxic environment: effects of submaximal exercise. Int J Environ Health Res. 2007;17:53-60.

60. Chen Y-CC, Lin F-CC, Shiao G-MM, Chang S-CC. Effect of rapid ascent to high altitude on autonomic cardiovascular modulation. Am J Med Sci. 2008;336: 248-253.

61. Zupet P, Princi T, Finderle Z. Effect of hypobaric hypoxia on heart rate variability during exercise: A pilot field study. Eur J Appl Physiol. 2009;107:345-350.

62. Cavaletti G, Moroni R, Garavaglia P, Tredici G. Brain damage after high-altitude climbs without oxygen. Lancet. 1987;1(8524):101.

63. Nelson TO, Dunlosky J, White DM, Steinberg J, Townes BD, Anderson D. Cognition and metacognition at extreme altitudes on Mount Everest. J Exp Psychol Gen. 1990;119:367-374.

64. Berry DT, McConnell JW, Phillips BA, Carswell CM, Lamb DG, Prine BC. Isocapnic hypoxemia and neuropsychological functioning. J Clin Exp Neuropsychol. 1989;11:241-251.

65. Botella de Maglia J, Garrido Marín E, Catalá Barceló J. Transient motor aphasia at high altitude [in Spanish]. Rev Clin Esp. 1993;193:296-298.

66. Lieberman P, Protopapas A, Reed E, Youngs JW, Kanki BG. Cognitive defects at altitude. Nature. 1994;372: 325.

67. Denison DM, Ledwith F, Poulton EC. Complex reaction times at simulated cabin altitudes of 5,000 feet and 8,000 feet. Aerosp Med. 1966;37:1010-1013.

68. Bolmont B, Thullier F, Abraini JH. Relationships between mood states and performances in reaction time, psychomotor ability, and mental efficiency during a 31-day 
gradual decompression in a hypobaric chamber from sea level to 8848 m equivalent altitude. Physiol Behav. 2000; 71:469-476.

69. Hornbein TF, Townes BD, Schoene RB, Sutton JR, Houston CS. The cost to the central nervous system of climbing to extremely high altitude. N Engl J Med. 1989; 321:1714-1719.

70. Li P, Zhang G, You HY, Zheng R, Gao YQ. Trainingdependent cognitive advantage is suppressed at high altitude. Physiol Behav. 2012;106:439-445.
71. Rea MS, Figueiro MG, Bullough JD, Bierman A. A model of phototransduction by the human circadian system. Brain Res Brain Res Rev. 2005;50:213-228.

72. Cajochen C, Münch M, Kobialka S, et al. High sensitivity of human melatonin, alertness, thermoregulation, and heart rate to short wavelength light. J Clin Endocrinol Metab. 2005;90. 1311-6.

73. Monk TH, Buysse DJ, Reynolds CF III, et al. Circadian rhythms in human performance and mood under constant conditions. J Sleep Res. 1997;6:9-18. 